\title{
Comparison of the superconducting rectangular critical fields with the same area at $\kappa=1.5$
}

\author{
Fuad Anwar ${ }^{1}$, C. Cari' ${ }^{2}$ Alqaan Maqbullah Ilmi $^{3}$ \\ ${ }^{1,2,3}$ Masters Program in Physics, Postgraduate Sebelas Maret University, J1. Ir. Sutami no 36 \\ Kentingan, Jebres Surakarta \\ Email: ${ }^{1}$ fanwar@staff.uns.ac.id
}

Received 12 July 2019, Revised 19 September 2019, Published 30 September 2019

\begin{abstract}
The Time Dependent Ginzburg-Landau (TDGL) equation can be used to study the characteristics of superconductors in the evolution of time to reach equilibrium. This study uses the $\Psi U$ method to numerically calculate critical field values more easily. Previous research has been carried out on size variations, kappa variations, proximity effects and so on. In this study, a comparison between squares and rectangles with the same size of area for type II superconductor, then it is found that the critical field $\mathrm{Hc}_{3}$ is the same value for each of the same area.
\end{abstract}

Keywords: type II-superconductor, critical field, TDGL equation, $\Psi U$ method

\section{Introduction}

Qiang (2005), Rosyida, et al (2017), and Anwar, et al (2014), have done numeric research to solve TDGL equation computationally. In the calculations, use $\psi U$ method to obtain consistency convergent results as shown by Winiecki and Adams (2002). In addition to the parameters $\kappa$ and size, the influence of the critical field is also derived from the Proximity Effect as shown by Anwar (2015), anisotropic perturbations as shown by Lin and Lipavský (2008), and the Bifurcation Effect as shown by Ma and Wang (2005), Anwary, et al (2018), and Belova, et al (2011), performed calculations for integer parameter $\kappa$ parameters to find an effect on critical field changes. Poskarina, et al (2018), made calculations using variations in size with rectangular shapes found that the greater the size affects the critical field. This research studies the value of type II superconducting critical fields comparing rectangular and square shapes with various size variations that have the same area at $\kappa=1.5$.

\section{Research Method}

The search for critical field values from the TDGL equation cannot be done analytically because the equation is complex and coupled so computational calculations are performed as shown by Saitoh and Hayakawa (2012). This study uses a squareshaped superconducting material with the size of the sides $N x$ and $N y$ then subjected to a uniform external magnetic field in the direction of the positive $z$-axis and also the time wave as shown in Figure 1. 


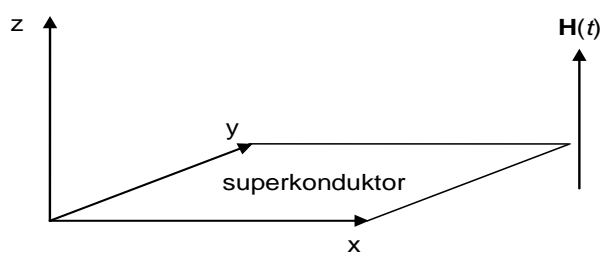

Figure 1. Superconducting schemes under study

The use of the $\psi U$ method means that superconducting materials are considered to be composed of a set of cells of a size, which for each cell contains three fundamental quantities namely $\psi_{i j}, U x$ and $U y$.

As shown in [12], [13], and [14], the TDGL Equation can be written as follows :

$$
\begin{gathered}
\frac{\partial \psi(\mathbf{r}, t)}{\partial t}=(\nabla-i \mathbf{A}(\mathbf{r}, t))^{2} \psi(\mathbf{r}, t)+\psi(\mathbf{r}, t)-|\psi(\mathbf{r}, t)|^{2} \psi(\mathbf{r}, t) \\
\sigma \frac{\partial \mathbf{A}(\mathbf{r}, t)}{\partial t}=\frac{1}{2 i}\left(\bar{\psi}(\mathbf{r}, t) \nabla \psi(\mathbf{r}, t)-\psi(\mathbf{r}, t) \nabla \bar{\psi}(\mathbf{r}, t)-2 i|\psi(\mathbf{r}, t)|^{2} \mathbf{A}(\mathbf{r}, t)\right)-\kappa^{2}(T) \nabla \times\left(\nabla \times \mathbf{A}(\mathbf{r}, t)-\mathbf{H}_{e x t}(\mathbf{r}, t)\right)
\end{gathered}
$$

With the terms of the parameters the default is

$$
\left(-i \hbar \nabla+\frac{e_{s}}{c} \mathbf{A}\right) \psi \cdot \mathbf{n}=0
$$

And the condition of the magnetic field limit is

$$
\mathbf{H}_{e x t}=\nabla \times \mathbf{A}
$$

The value of the squared obtained TDGL equation and the boundary conditions can be technically observed from the value of the ratio of the circumference and area of the superconductor. The circumference ratio value per area close to zero shows that the boundary condition equation does not have much effect so that for this size it can be categorized as a large bulk.

\section{Results and Discussion}

The value of the size variation used in this study is presented in the following table 1:

Table 1. Size Variation Value

\begin{tabular}{cccccccc}
\hline Number & Wide & Long & Large & Shape & $H c_{1}$ & $H c_{3}$ & $\begin{array}{c}\text { Circumference } \\
\text { per Area }\end{array}$ \\
\hline 1 & 12 & 12 & 144 & Square & 0.9 & 2,4 & 0.33 \\
& 9 & 16 & 144 & Rectangle & 0.9 & 2.5 & 0.35 \\
& 8 & 18 & 144 & Rectangle & 1 & 2.5 & 0.36 \\
& 6 & 24 & 144 & Rectangle & 1.5 & 2.7 & 0.42 \\
2 & 16 & 16 & 256 & Square & 0.7 & 2,4 & 0.25 \\
3 & 8 & 32 & 256 & Rectangle & 1 & 2.5 & 0.31 \\
& 32 & 32 & 1024 & Square & 0.6 & 2,3 & 0.13 \\
& 16 & 64 & 1024 & Rectangle & 0.6 & 2,4 & 0.16 \\
& 8 & 128 & 1024 & Rectangle & 1 & 2.5 & 0.27 \\
\hline
\end{tabular}

Table 1 shows that a small increase in circumference value per area did not change the value of $\mathrm{Hc}_{1}$. This can be seen in the circumference value of 0.33 with 0.35 and 
0.13 with 0.16 . As it is known that the circumference represents the physical properties of superconductors from the calculation of boundary conditions while the area represents the physical properties of superconductors from TDGL calculations. So that it can practically study the physical properties of superconductors through a comparison of circumference and area.

$\mathrm{Hc}_{1}$ is a low critical field where the external field begins to penetrate superconducting material in the form of vortex. This shows that the external magnetic field has succeeded in penetrating some of the material but the other part is still superconducting, or also known as a mixed state. Whereas $\mathrm{Hc}_{3}$ is an external field value that is able to totally eliminate superconducting properties.

Suzuki (2005), explains that $\mathrm{Hc}_{1}$ can be sought when a turning point occurs in a squared, ie when the psi squared value first shows an increase. Meanwhile, to find $\mathrm{Hc}_{3}$ by finding an external magnetic field where psi squared is zero at first. Cyrot and Pavuna (1992), show that this condition occurs when the material has totally lost its superconducting properties. Here is a graph of squares divided into small, medium and large areas.

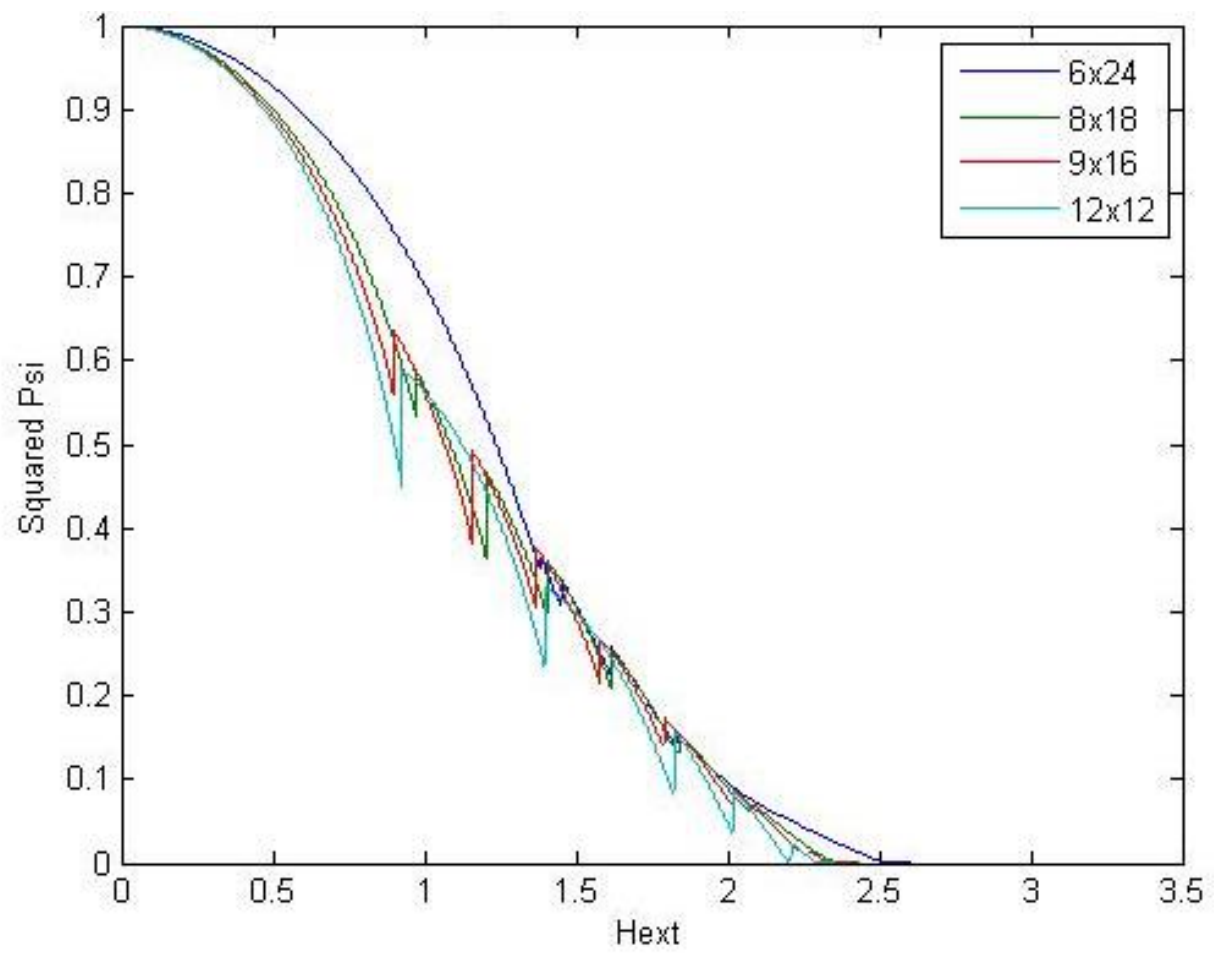

Figure 2 . Critical field values for small area sizes 


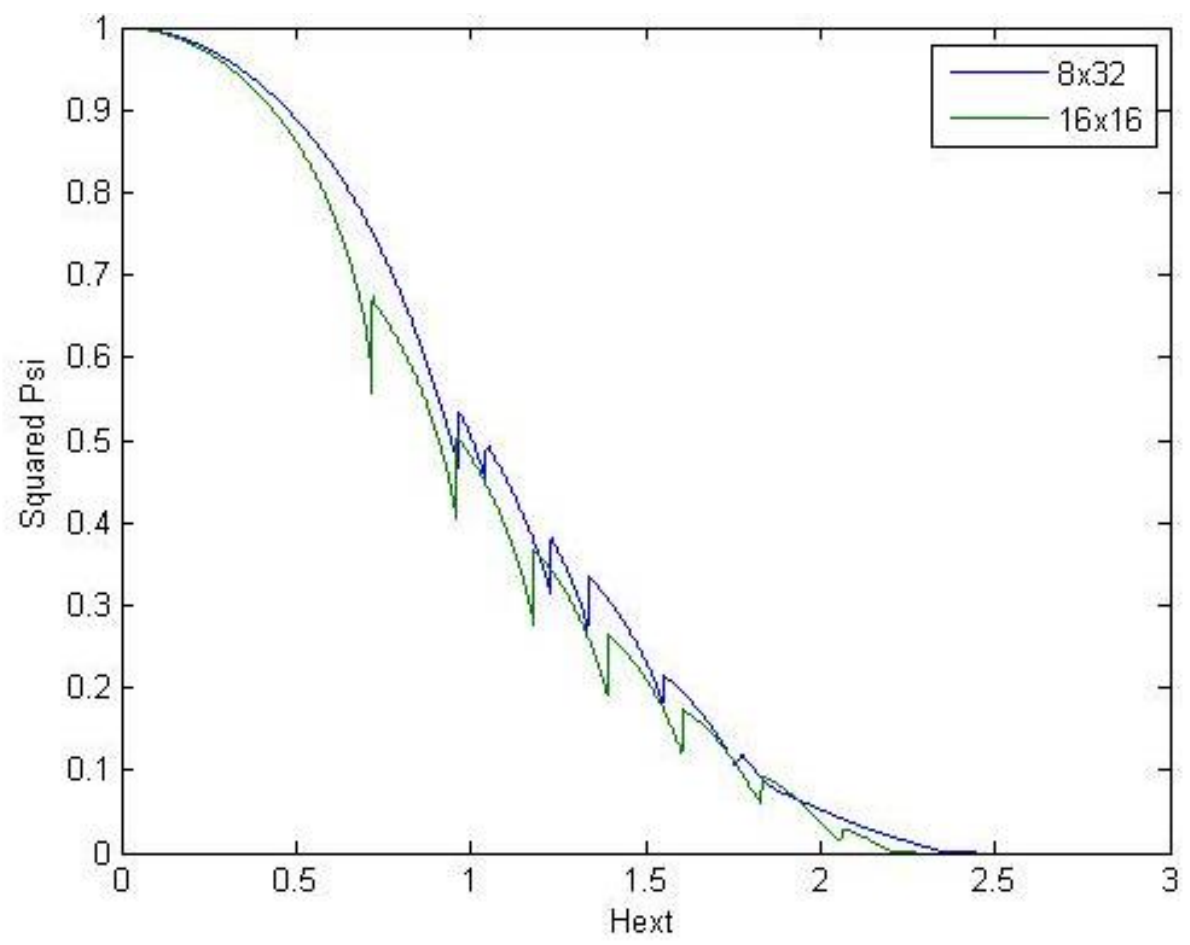

Figure 3 . Critical field values for medium size

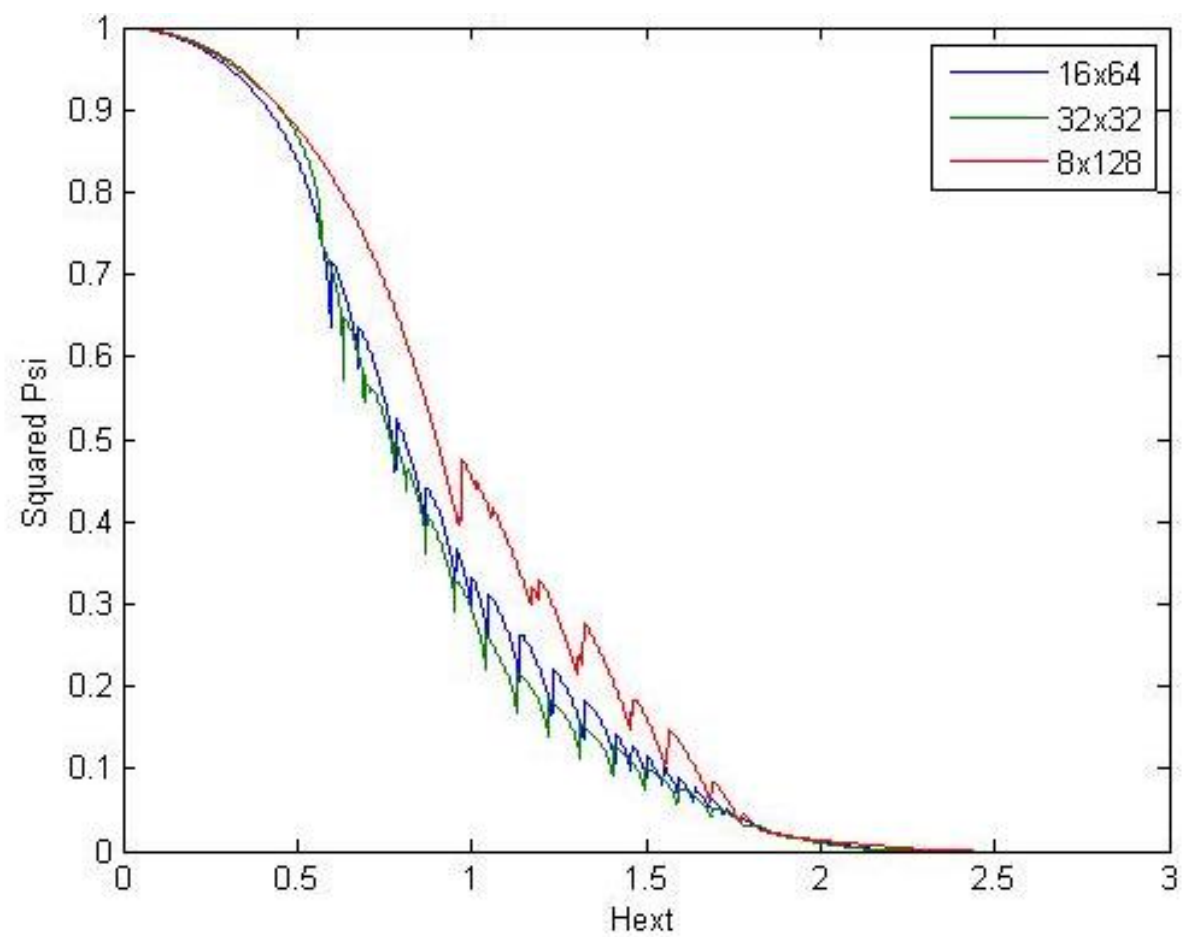

Figure 4. Critical field values for large area sizes

The graph shown in Figure 2-4 shows that in general the pattern shown is the same as the theory of superconducting behavior when given an external magnetic field. Then look for the critical field by tracing from the computational output data. Figure 5-7 shows that the value of the critical field tend almost stable with a decreased less when 
the width is enlarged. An interesting equation is that the critical field value for a square is always smaller than the long square with the same area.

\section{small size}

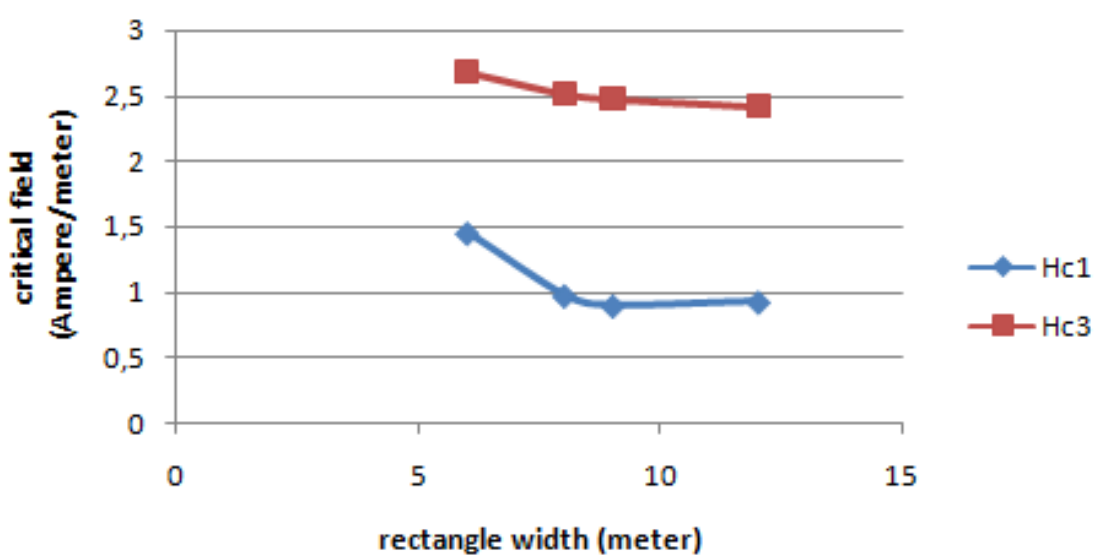

Figure 5 . Critical field values for small sizes

medium size

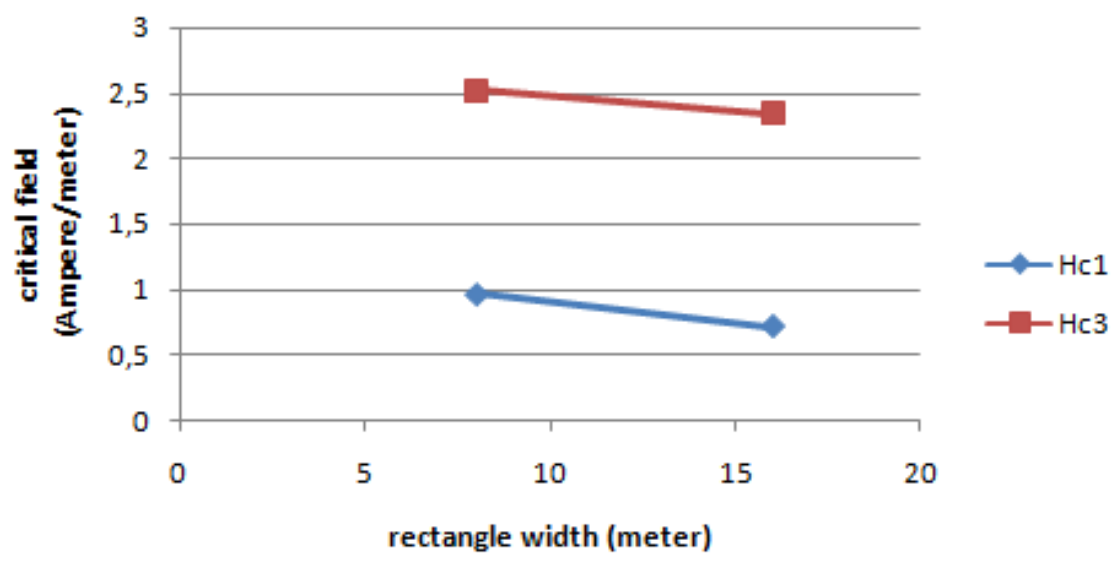

Figure 6 . Critical field values for medium sizes

\section{big size}

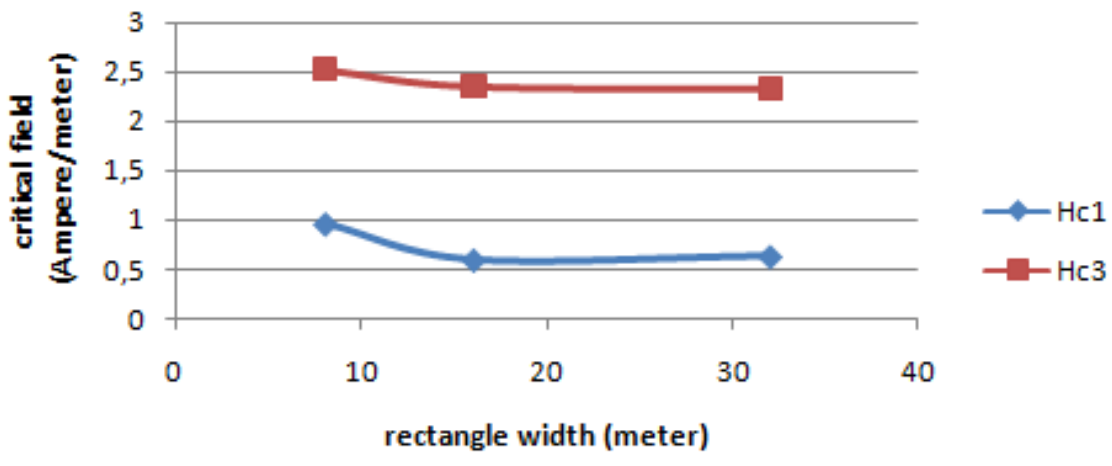

Figure 6. Critical field values for large sizes 
Figure 5-7 shows that the value of the critical field tends to be almost stable with a smaller decrease when the width is enlarged. The results were interesting contained in the value of the critical field for a square is always smaller than the square panja $\mathrm{n} g$ the same area. Kopnin (2006), and Tinkham (1996), say that the effect of size variations on the value of this critical field is related to the depth of penetration of the external field to the material.

\section{Conclusion}

The critical field values of the superconductors of type II for $\kappa=1.5$ have been calculated successfully to compare the critical field values between square and rectangular shapes with the same area of 144, 256 and 1024 using the numerical computation of the $\psi U$ method. Type II superconductors have two critical fields namely low critical field $\mathrm{Hc}_{1}$ and surface critical field $\mathrm{Hc}_{3}$. The value of $\mathrm{Hc}_{1}$ and $\mathrm{Hc}_{3}$ depends on the shape with a circumference ratio value per area.

\section{Acknowledgements}

This research would not have been possible without the support of the Graduate Program of Sebelas Maret University.

\section{References}

Qiang Du, Numerical approximations of the Ginzburg - Landau models for superconductivity. Journal of Mathematical Physics, 46, pp. 1-22, 2005. https://doi.org/10.1063/1.2012127

R Rosyida, F. Anwar, and Darmanto. Numerical simulation of dimension effects on critical fields of rectangular superconductors. International Conference on Science and Applied Science, pp. 1-5, 2017. https://iopscience.iop.org/article/10.1088/1757-899X/179/1/012008/pdf

F. Anwar, P. Nurwantoro, and A. Hermanto. Critical Terrain Study on Completion of the Ginzburg-Landau Equation of the Time Gaze. Proceedings of the XXVIII Scientific Meeting of Central Java \& Yogyakarta HFI, Yogyakarta, pp. 145148, 2014.

T. Winiecki and CS Adams. A Fast Semi-Implicit Finite-Difference Method for the TDGL Equations. Journal of Computational Physics, 179, pp. 127-139, 2002. https://doi.org/10.1006/jcph.2002.7047

F. Anwar. Model Study: Effect of Proximity and Non-Isotropic Effects on the Type II Mesoscopic Superconducting Magnetic Properties. Doctoral dissertation. UGM, Yogyakarta, 2015.

Pei-Jen Lin and P. Lipavský, Gradient corrections to the time-dependent GinzburgLandau equation for anisotropic perturbations of quasiparticles, Physical Review B, vol. 77, pp. 1-16, 2008.

T. Ma and S. Wang, Bifurcation and stability of superconductivity, Journal of Mathematical Physics, vol. 46, pp. 1-31, 2005. https://doi.org/10.1063/1.2012128 
R. Anwary, F. Anwar and H. Purwanto. Study the effect of area variations on rectangular Type II superconducting properties on the state of the proximity effect. Proceedings of the Quantum National Seminar, vol. 25, pp. 724-731, 2018.

P. Belova, K. B. Traito, and E. Lähderanta, Eilenberger and Ginzburg-Landau models of the vortex core in high אsuperconductors, Journal of Applied Physics, vol. 110, pp. 1-7, 2011.

N. Poskarina, F. Anwar, and AD Sutomo, Study on the Effects of Ginzburg-Landau Parameters on Rectangular-Shaped Type II Critical Superconductor Fields, Proceedings of the Quantum National Seminar, vol. 25, pp. 699-704, 2018.

Kuniyasu Saitoh, and Hisao Hayakawa, Time dependent Ginzburg-Landau equation for sheared granular flow, AIP Conference Proceedings, vol. 1501, pp. 1001$1008,2012$.

M. Suzuki and IS Suzuki, Lecture Note on Solid State Physics Ginzburg-Landau Theory for Superconductivity, Newyork,: State University of Newyork, 2007.

Mads Peter Sørensen, Niels Falsig Pedersen, and Magnus Ögren, The dynamics of magnetic vortices in type II superconductors with pinning sites studied by the time dependent Ginzburg-Landau model, Physica C: Superconductivity and its applications, vol. 533, pp. 40-43, 2017

Buyang Li, and Zhimin Zhang, A new approach for numerical simulation of the timedependent Ginzburg-Landau equations, Journal of Computational Physics, vol. 303, pp. 238-250, 2015.

Jorge Berger, Time-dependent Ginzburg-Landau equations with charged boundaries, Journal of Mathematical Physics, vol. 46, pp. 1-14, 2005

M. Cyrot and M. Pavuna. Introduction to Superconductivity and High Tc Materials, World Scientific Publication co. Ptc. Ltd., Singapore, 1992.

NB Kopnin, Theory of Superconductivity, Helsinki University of Technology, 2006.

M. Tinkham, Introduction to Superconductivity, Singapore,: McGraw-Hill Inc., 1996. 